\title{
Problemas laborales asociados al consumo de alcohol
}

\author{
BASTIDA, N. \\ Médico de Familia del CAP Dr. Sayé (Raval Nord) Barcelona. \\ Médico de Empresa de la empresa Dupont Ibérica, S.L.
}

Enviar correspondencia: Nuria Batida Bastús. C/ Córcega, 254, 4, 1ª Barcelona 08008. E-mail: 13930nbb@comb.es

\section{RESUMEN}

Los problemas derivados del consumo de alcohol en la población trabajadora son muy importantes. Estudios realizados en numerosos países demuestran que más del $70 \%$ de los consumidores de drogas y alcohol trabajan. La población más bebedora es la masculina, de edad comprendida entre los 25 y los 44 años. La prevalencia del consumo de alcohol en la población trabajadora supera a la de la población general. En España, alrededor de un 24\% de los trabajadores consume una cantidad de alcohol que es considerada de riesgo potencial para la salud física. Desde los servicios de salud laboral se atribuye a las drogas (alcohol) muchos efectos negativos entre los que destaca, el mayor absentismo, incremento de las bajas laborales, mayor accidentabilidad (en nuestro país, alrededor del $25 \%$ de los accidentes laborales tienen como causa básica el alcohol), disminución del rendimiento y mayor conflictividad (entre el 15-40\% de sanciones disciplinarias se relacionan con el consumo de alcohol).

Los servicios de Salud Laboral son, junto a la Atención Primaria, el eje básico para la prevención de los problemas relacionados con el alcohol. En todas las empresas deberían existir "Programas de Ayuda al Empleado" (PAE), llevados a cabo generalmente, por profesionales externos y coordinados con los servicios médicos de la compañía y cuyo objetivo es la pronta identificación y resolución de problemas que pueden interferir en la vida personal y/o laboral de los empleados y familiares manteniendo la máxima confidencialidad.

Palabras clave: alcohol, problemas laborales, consumo, prevención, empresas.

\section{SUMMARY}

Alcohol related problems in the working population are very important. Studies that have taken place in many countries show that above $70 \%$ of alcoholics and drug users work. Most of the drinking population are men aged 25 to 45 .

The prevalence of alcohol consumption in the working population exceeds that of the general population. A survey exclusively done on the working population states that $24 \%$ of workers consume a quantity of alcohol that is considered a potential risk to physical health. From the workers Health Services perspective, alcohol is seen as a major cause of concern: absenteeism, an increase of sick leave, accidents (25\%), deterioration of performance and an increase of behavioural problems (between 15-40\% of disciplinary sanctions are related to alcohol consumption) are the most prevalent alcohol related problems at work.

The Occupational Health Services are, together with Primary Health Care Centres the basic key to the prevention of alcohol-related problems. All companies should have "Employee Assistance Programs" (EAP), led by external professionals and coordinated with the company's medical services. The objective should be early identification of problems which can interfere with personal or working conditions of employees and their families maintaining the maximum confidentiality.

Key words: alcohol, work problems, consumption, prevention, enterprises. 


\section{EL CONSUMO DE BEBIDAS ALCOHÓLI- CAS EN EL ÁMBITO LABORAL}

E alcoholismo es uno de los problemas sanitarios de mayor relevancia mundial, con una elevada prevalencia en la población española, y con unos costes sociales y económicos que superan el medio billón de pesetas/año'.

España ocupa el primer lugar dentro de la Unión Europea en oferta y accesibilidad al consumo de bebidas alcohólicas, con un establecimiento de dispensación de bebidas alcohólicas (bar o equivalente) por cada 169 habitantes. España es además el séptimo país del mundo en cuanto a consumo per cápita, con 10.4 litros por habitante y año en 1991. En general, el alcohol es responsable de alrededor de 12.000 fallecimientos al año, lo que supone cerca del $4.5 \%$ de la mortalidad total, y de un considerable número de años de vida potenciales perdidos. El coste social del consumo de alcohol supone alrededor de medio billón de pesetas/año en el conjunto del estado español, debido sobre todo a enfermedad, accidente laboral y absentismo².

Estudios realizados en numerosos países demuestran que el $71 \%$ de los 'consumidores problemáticos de drogas y alcohol' trabajan. ${ }^{3}$ Un reciente estudio EDIS/ FAD ${ }^{4}$ indica como problemas de salud prioritarios en el mundo laboral el tabaco y las bebidas alcohólicas.

El alcohol además de ser una sustancia tóxica en sí misma, contribuye a aumentar el efecto de numerosas sustancias tóxicas, aún siendo ingerido en pequeñas cantidades.

Este fenómeno se conoce como "sinergismo" entre el alcohol y algunos tóxicos industriales como la Cianamida cálcica o los hidrocarburos clorados presentes en actividades de desengrase, sector metalúrgico, industria de relojería..., etc.

La cultura mediterránea, vitivinícola, ha creado mecanismos que permiten una "tolerancia social" frente a los conflictos generalizados por el uso de las bebidas alcohólicas, que a su vez es responsable de la ocultación, en los medios laborales, de la existencia de problemas e incluso de accidentes asociados a su consumo.

La repercusión social del paciente alcohólico es alta. Aunque la figura más representativa del paciente alcohólico sea la del vagabundo en estado de embriaguez, esta clase de pacientes representan únicamente alrededor del $5 \%$ de los alcohólicos. La inmensa mayoría viven con sus familias, tienen trabajo, y llevan una vida aparentemente normal ${ }^{5}$.

La población más bebedora es la masculina, de edad comprendida entre los 25 y los 44 años. Por dicho motivo, la prevalencia del consumo de alcohol en la población trabajadora supera a la de la población general. Cabe constatar que la mayoría de los pacientes en tratamiento por dependencia alcohólica o con antecedentes de problemas superados se encuentra en una situación laboral activa. ${ }^{6}$

Los datos de la encuesta EDIS/ UGT $1987^{7}$ que se refieren exclusivamente a la población trabajadora permiten afirmar que un $24,1 \%$ (prácticamente uno de cada cuatro) de los trabajadores consume una cantidad de alcohol que es considerada de riesgo potencial probado para la salud física $(76 \mathrm{ml}$ de alcohol puro /día 61g); un $16 \%$ supera los $100 \mathrm{ml} / 80 \mathrm{~g}$; un $8.7 \%$ consume $150 \mathrm{ml} / 120 \mathrm{~g}$ y un $7{ }^{\prime} 5 \%$ supera dicha cantidad, sobrepasando los $150 \mathrm{~g}$ y llegando a superar los $200 \mathrm{~g} /$ día de alcohol puro.

La encuesta EDIS/ FAD ${ }^{4}$ del 1996 señaló que el $81.2 \%$ de los trabajadores ocupados consumieron alcohol en el último mes y un $58 \%$ la última semana. El $14.4 \%$ indicó haber consumido el día anterior por encima de la cantidad de riesgo $(75 \mathrm{ml}$ de alcohol puro / día). El consumo de alto riesgo tiene lugar diaria o regularmente en el $9.2 \%$ de trabajadores ocupados, admitiendo el $2 \%$ que bebe en el lugar de trabajo. (Figura 1)

En la misma encuesta se detecta que la población trabajadora consume más alcohol que la población general, y los parados consumen todavía mayor cantidad, en todas las edades, que los trabajadores ocupados (Tabla $1)^{5}$ 
Figura 1. Características del consumo de alcohol en los trabajadores ocupados.

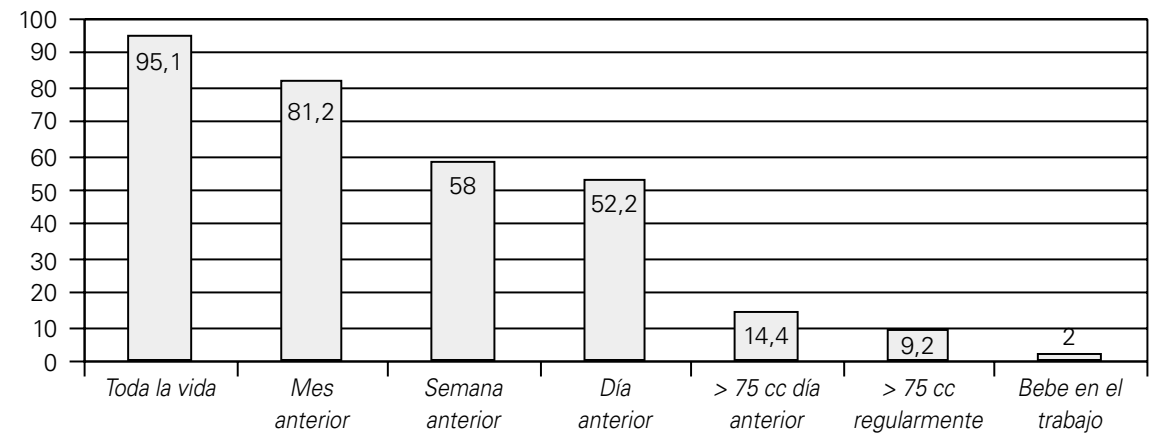

Fuente: EDIS/FAD, 1996

\section{Tabla $1^{5}$}

\begin{tabular}{lccc}
\hline Patrón de consumo & Población general* & Ocupados* & En paro** \\
\hline Han probado & $90,2 \%$ & $95,1 \%$ & $96,7 \%$ \\
Consumo último mes & $53,3 \%$ & $81,2 \%$ & $77,9 \%$ \\
Consumo última semana & $41,2 \%$ & $58,0 \%$ & $55,1 \%$ \\
Consumo día anterior & - & $52,2 \%$ & $44,0 \%$ \\
\hline
\end{tabular}

*Fuente: CIS /DGPNSD, 1993.

**Fuente: EDIS /FAD, 1996.

El $14,4 \%$ de los trabajadores ocupados refiere haber consumido más de $75 \mathrm{cc}(60 \mathrm{~g})$ el día anterior, lo que implica riesgo elevado; el $20,6 \%$ consumió entre $26-75 c c$, presentando una ingesta de riesgo entre ligero y moderado (Figura 2) ${ }^{4}$.

Es importante destacar las variables en función del nivel profesional y socioeconómico: la frecuencia de bebedores por encima de $75 \mathrm{cc}$ de alcohol / día en la muestra estudiada es mayor cuanto menor es el nivel sociocultural (Figuras 3 y 4). Al analizar el sector productivo, destaca la mayor concentración de bebedores abusivos en sectores de riesgo como la construcción $(26,9 \%)$, el transporte y comunicaciones $(19,5 \%)$ y el sector de la minería, energía y químico $(16,7 \%)^{4}$.

Es de destacar la alta tasa de siniestralidad laboral que por las características del trabajo se produce en el sector de la construcción y donde el consumo de bebidas alcohólicas durante la jornada laboral o en las horas previas a la misma, incluso en dosis bajas, produce modificaciones conductuales que pueden interferir con la realización de las tareas propias de cada trabajo y ocasionar situaciones de especial riesgo.

Un estudio realizado por Webb et $\mathrm{al}^{8}$, demostró que los trabajadores que presentaban problemas relacionados con el alcohol, según el test de Mortimer-Filkins ${ }^{9}$ tenían 2.7 más de ausencias en el trabajo por accidentes que los trabajadores que no tomaban alcohol ${ }^{10}$.

\section{FACTORES QUE FAVORECEN EL CON- SUMO DE ALCOHOL}

Los propios trabajadores no suelen atribuir al trabajo un papel significativo en el desarro- 
Figura 2. Distribución del consumo de alcohol puro en trabajadores ocupados.

Fuente: EDIS/FAD, 1996
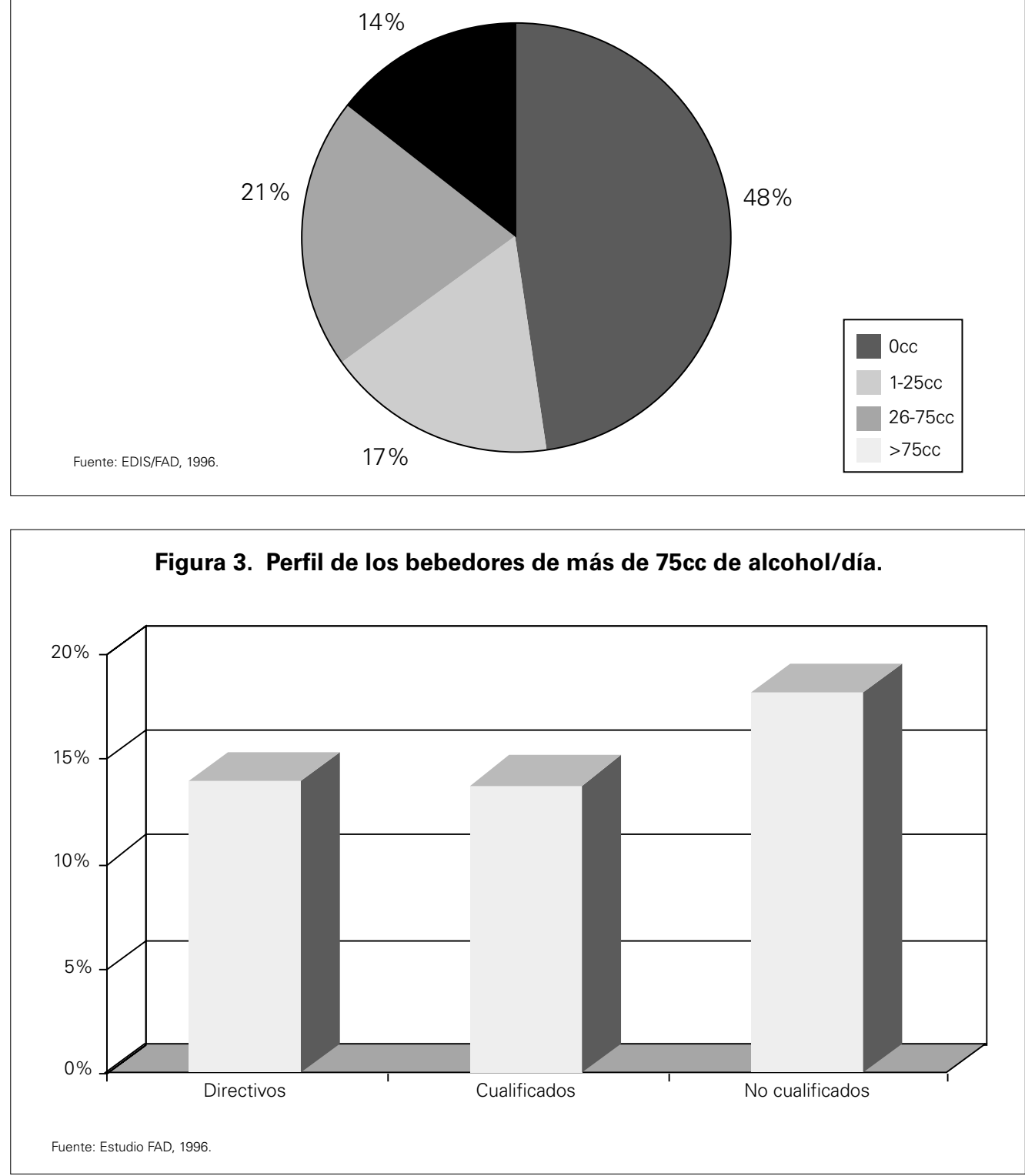

llo de problemas de alcohol propios o ajenos ${ }^{4}$ (Figura 5). Sin embargo multitud de estudios epidemiológicos reconocen que la salud está relacionada con las vivencias en el medio laboral. Este reconocimiento es manifiesto en las normativas vigentes en el estado español, donde se recogen los aspectos relacionados con la organización del trabajo, condiciones del trabajo y relaciones sociales, como factores influyentes en la salud global del individuo, introduciéndose el concepto de "factores psicosociales" en numerosa documentación téc- 
Figura 4. Perfil de bebedores de más de 75cc de alcohol/día según el nivel socioeconómico.

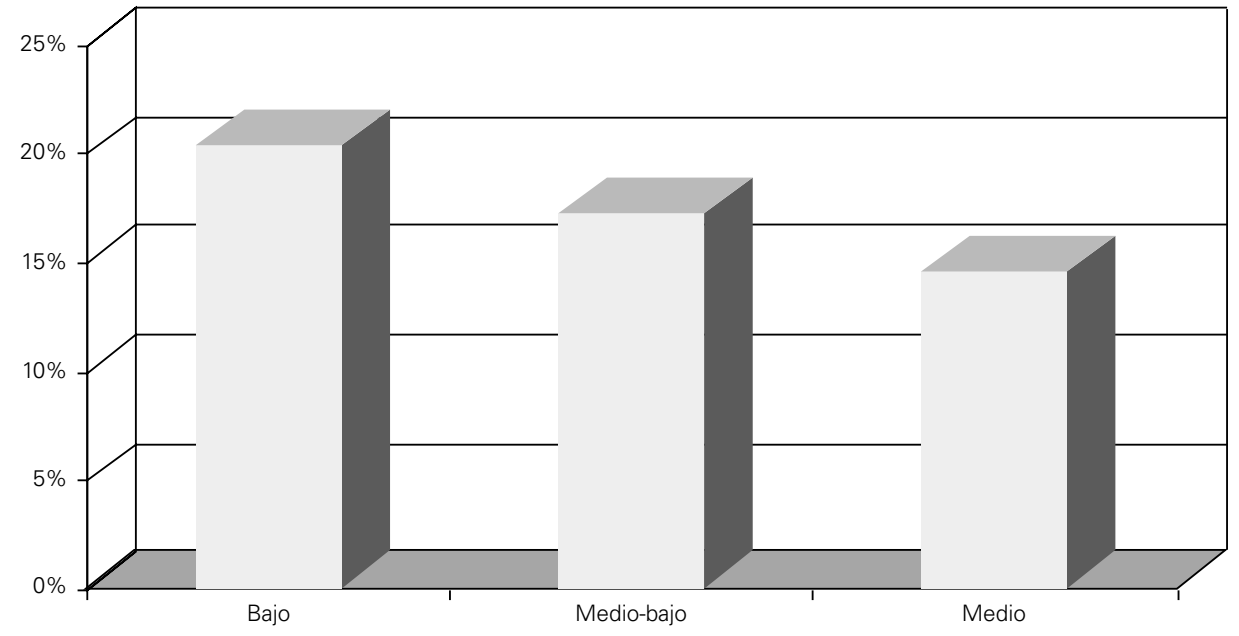

Fuente: Estudio FAD, 1996

Figura 5. Motivos aducidos por los bebedores para su consumo de alcohol.

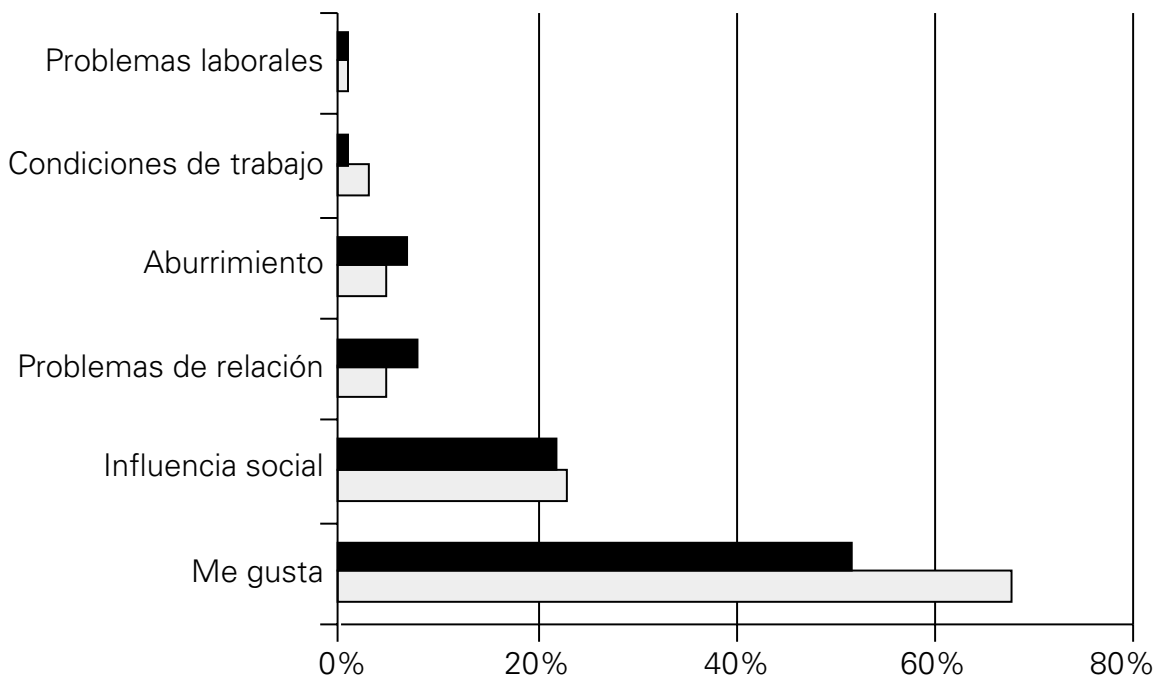

Fuente: EDIS/FAD, 1996.

nica editada por el Instituto Nacional de Seguridad de Higiene en el Trabajo.
Existen una serie de elementos del ambiente laboral o características del puesto 
de trabajo que, sin tener una relación causa efecto, pueden favorecer el inicio y mantenimiento de un consumo excesivo o inadecuado de bebidas alcohólicas. No está muy claro, sin embargo, por qué los problemas relacionados con el alcohol son distintos según los trabajos y las personas. La disponibilidad física y social de alcohol en el lugar de trabajo son dos factores que ayudarían a explicar estas diferencias. ${ }^{8}$

\subsection{Se han descrito una serie circunstancias en el medio laboral que incrementan la oferta y disponibilidad del alcohol.}

- Pautas del colectivo social (ambientes artísticos, fuerzas armadas, gente de mar...)

- Relaciones públicas (vendedores, representantes de comercio, hombres de negocios....)

- Disponibilidad de alcohol (camareros, cocineros...)

- Ausencia de normas sobre el consumo de bebidas alcohólicas en el lugar de trabajo.

\subsection{Circunstancias que favorecen el con- sumo utilitario.}

- Condiciones materiales y físicas del trabajo:

- elevadas temperaturas

- horarios prolongados

- rotación horaria (provoca alteraciones del ritmo circadiano del sueño, digestivas, aumenta el nivel de ansiedad entre los padres y los hijos ${ }^{11}$ se asocia con alteraciones de la atención y del tiempo de reacción ${ }^{12}$. Algunos estudios han demostrado que los empleados que tienen turnos de tarde o de noche consumen más alcohol. También lo toman para dormir durante el día ${ }^{13}$.

- toxicidad ambiental

- trabajo a la intemperie

- esfuerzo físico (construcción, minería, turnos de noche, pesca...)

- Condiciones psicosociales estresantes
- objetivos poco claros en lo que respecta al desarrollo de su carrera profesional (carencia de perspectivas de promoción)

- excesiva carga de trabajo o responsabilidad

- trabajo excesivamente automatizado (monótono) o precario y sin capacidad de control de fallos

- inseguridad en el empleo

- conflictividad laboral

- aislamiento social o comunicación social difícil (mezcla de idiomas y razas, en tripulaciones de barco...)

- malas relaciones entre la dirección y los empleados

- condiciones laborales inadecuadas

- un empleo que exija alta movilidad (viajes continuos, separación de la familia)

El estrés resulta de un proceso complejo de interacción entre ciertos factores estresores externos y las correspondientes reacciones individuales de respuesta. En el trabajo, las condiciones psicosociales capaces de actuar como estresores tienen también un papel preponderante en el síndrome de "estar quemado" (burn-out), considerado como una respuesta inadecuada al estrés emocional crónico.

\section{REPERCUSIONES LABORALES DEL CON- SUMO DE ALCOHOL}

Los efectos nocivos del consumo de alcohol en el lugar de trabajo y sobre el rendimiento laboral son frecuentes a todos los niveles. El alcoholismo es una enfermedad escondida, con problemas de comportamiento importantes que, por lo general, no se atribuyen a su verdadero origen.

El alcohol y las drogas son considerados un importante problema por alrededor de la mitad de los trabajadores $(51,6 \%$ de los ocupados y $45 \%$ de los parados) ${ }^{4}$. La razón que más se nombra para considerar dichos consumos como problema es la disminución de la productividad o rendimiento $(25,5 \%$ de los trabajadores activos y el $26,1 \%$ de los trabajadores en paro) seguida de la provocación de 
accidentes $(23,4 \% \text { y } 22,6 \%)^{7}$. Aunque sólo el $2 \%$ reconoce consumir en el lugar de trabajo, las repercusiones sobre el mismo alcanzan unas dimensiones considerables (Figura 6): el $8.5 \%$ indica haber tenido algún problema de relación interpersonal, el $7.1 \%$ de los trabajadores en activo declara problemas de salud, el $3.1 \%$ de absentismo en relación con la bebida y el $2.8 \%$ cree haber disminuido el rendimiento laboral. El $0.6 \%$ relata accidentes de traba- jo y el $1.6 \%$ accidentes de tráfico. En los trabajadores activos, los problemas laborales atribuibles al alcohol ocupan el segundo puesto, tras los de salud en general ${ }^{4}$.

Desde los servicios de salud laboral, se atribuye a las drogas una pluralidad de efectos negativos sobre el mundo laboral, entre los que destaca el absentismo por enfermedad o por accidente y las ausencias y retrasos injustificados (Tabla II).

Figura 6. Consecuencias del consumo de alcohol en trabajadores ocupados.

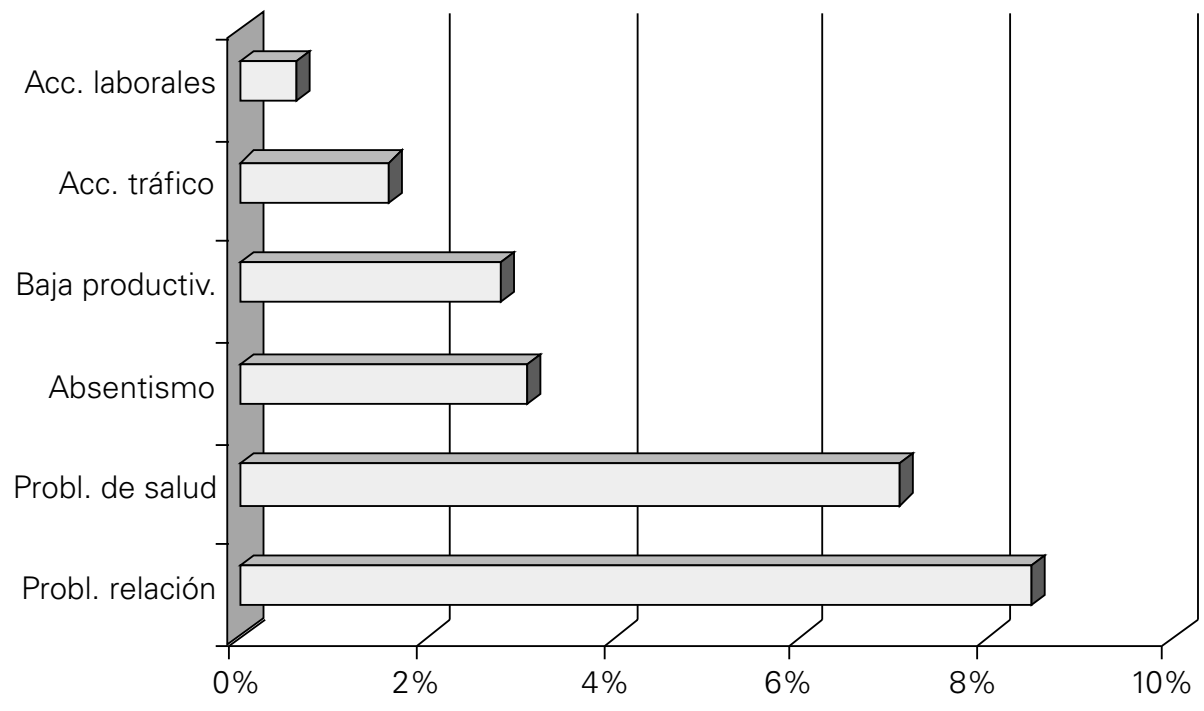

Fuente: EDIS/FAD, 1996.

Tabla II. Efectos atribuidos al consumo de drogas por los servicios de Salud Laboral.

\section{EFECTOS}

Mayor absentismo

Incremento de las bajas laborales

Mayor accidentabilidad

Disminución del rendimiento

Mayor conflictividad

Incumplimiento de la jornada laboral

Comisión de más errores

Aumento de expedientes disciplinarios

Incremento del número de despidos
$\%$ DE SERVICIOS DE SALUD LABORAL

$75.0 \%$

$58.3 \%$

$50.0 \%$

$50.0 \%$

$50.0 \%$

$41.7 \%$

$33.3 \%$

$33.3 \%$

$33.3 \%$

Fuente: Sánchez Pardo. $1994^{14}$ 
Cinco años después de que se aprobara la Ley de Prevención de Riesgos Laborales, la siniestralidad laboral sigue siendo un problema creciente. Lejos de disminuir, las muertes por accidentes laborales han crecido en el año 2000 un 2\%, hasta cobrarse 1.133 vidas. El conjunto de siniestros se situó en 935.359, lo que supone un aumento del 7.6\% (Tabla III).

\begin{tabular}{|lccc|}
\hline \multicolumn{4}{|c|}{ Tabla III. Accidentes con baja en el puesto de trabajo en periodo } \\
enero/diciembre \\
1999/2000. \\
\hline 1999 & $\mathbf{2 0 0 0}$ & Var. \% \\
\hline AGRARIO & 47.152 & 44.113 & $-6,4$ \\
INDUSTRIA & 265.054 & 273.679 & 3.3 \\
CONSTRUCCIÓN & 207.673 & 232.078 & 11,8 \\
SERVICIOS & 347.893 & 385.489 & 10,8 \\
TOTAL & 867.772 & 935.359 & 7.8 \\
\hline Fuente: Departamento Confederal de Salud Laboral de CC. OO. ${ }^{15}$ & \\
\hline
\end{tabular}

Un estudio realizado por Geneviere et al ${ }^{16}$ sobre la ingesta de alcohol, la resaca posterior y los problemas en el trabajo, en una planta industrial a 832 empleados $(88 \%$ hombres), encontró que el hecho de beber durante el trabajo o acudir a trabajar con resaca post-ingesta tenia importantes implicaciones en la productividad, la seguridad laboral y ocasionaba frecuentes discusiones con los compañeros y supervisores

Los efectos y consecuencias del consumo de alcohol y otras drogas en el lugar de trabajo pueden ilustrarse mejor, según la Organización Internacional del Trabajo, por medio de los siguientes resultados:

- El número de accidentes del trabajo entre personas alcohólicas es entre dos y tres veces mayor que los que padecen los demás trabajadores. El alcohol y otras drogas serían responsables de entre un 15$30 \%$ del total de los accidentes del trabajo.

- En nuestro país, alrededor del 25\% de todos los accidentes laborales tienen como causa básica el alcohol. Entre el 20-25\% de los accidentes laborales afectan a personas en estado de intoxicación. Tales accidentes comprenden auto lesiones, heridas a terceros inocentes y daños al equipo.

- Las bajas laborales y el absentismo se llegan a triplicar. La frecuencia de interrup- ciones en el trabajo es 1.4 veces mayor a la del resto de los trabajadores.

- Entre el 15-40\% de los casos de sanciones disciplinarias aplicadas a trabajadores se relacionan con el consumo de drogas o alcohol.

- El gasto sanitario y social en concepto de atención médica, bajas e invalideces, así como de jubilación anticipada es entre el doble y el triple que en el resto de los trabajadores.

Como resultado extraíble de los estudios realizados, podemos resaltar los siguientes aspectos:

1. El consumo de alcohol se correlaciona positivamente con el número y la gravedad de los accidentes laborales. A mayor consumo de alcohol mayor riesgo de accidente.

2. Los accidentes de trabajo no sólo son más frecuentes en los bebedores sino que su recuperación es más prolongada y los costes más elevados.

\section{PAPEL DE LOS SERVICIOS MEDICOS DE EMPRESA}

Los problemas relacionados con el consumo de drogas y alcohol son tantos y tan 
diversos que las estrategias y soluciones han de adaptarse a las circunstancias particulares de cada empresa. Tanto el ambiente como los procesos de trabajo influyen en el consumo de drogas y alcohol, pero debe tenerse en cuenta la intervención de otros factores tales como la personalidad del trabajador, las actividades en el tiempo libre, las circunstancias familiares y la forma de vida.

Los servicios de Salud Laboral son, junto a la Atención Primaria, el eje básico para la prevención de los problemas relacionados con el alcohol, habida cuenta la elevada prevalencia de consumidores de riesgo entre la población laboral activa.

La ley 31/95 de Prevención de Riesgos Laborales, recoge la obligación del empresario de conseguir una protección eficaz de la Seguridad y Salud de los trabajadores frente a los riesgos inherentes al puesto de trabajo.

Los Servicios de Salud Laboral reúnen y analizan datos de Contingencias Laborales (accidentes de trabajo y/o enfermedades profesionales), Contingencias Comunes (accidentes no laborales y enfermedad común, relacionada o no con el trabajo) y los Exámenes de Salud previos y periódicos que se practican a los trabajadores. También forman parte del Comité de Seguridad y Salud de la Empresa, evaluando entre otras las condiciones ambientales de la empresa y del puesto de trabajo y participando en la formación higiénico-preventiva de los trabajadores.

Por lo que respecta a los problemas relacionados con el consumo de alcohol, el Medico de Empresa esta implicado tanto en la prevención primaria como la secundaria y terciaria en función de la política sanitaria de la empresa.

La prevención primaria se realizará a los abstemios o bebedores ligeros ( $<14$ UBEs/semana, en la mujer y 21 UBEs/semana, en el hombre). El mundo empresarial es un lugar idóneo para realizar prevención primaria por:

- La homogeneidad de la población,

- La globalidad (son asequibles todos los empleados),
- La continuidad de actuación a lo largo de tiempo que tales actuaciones permiten ${ }^{14}$.

La prevención secundaria y terciaria se realiza en los empleados que consumen $>28$ UBEs/semana en el hombre y >de 17 UBEs/semana en la mujer o $>$ de 10UBEs/24 horas. También en este tipo de prevención, el mundo empresarial tiene un importante papel debido a:

- La posibilidad de hacer detección precoz o diagnosticar patologías, puesto que la mayoría de bebedores excesivos se hallan en situación laboral activa

- El seguimiento de los casos detectados.

- El doble papel que puede jugar el puesto de trabajo: motivación para el tratamiento por el miedo a perder el trabajo y rehabilitador, a la hora de la recuperación, coordinándose con servicios específicos externos para controlar la evolución del proceso terapéutico y la reinserción laboral del enfermo, previa valoración de la idoneidad de su puesto de trabajo.

En la tabla II se expone esquemáticamente los tres niveles preventivos comentados anteriormente ${ }^{13}$.

En todas las empresas deberían existir " Programas de Ayuda al Empleado"(PAE). Los PAE son programas de ayuda llevados a cabo, generalmente, por profesionales externos a la compañía, coordinados con el Servicio Médico, y cuyo objetivo es la pronta identificación y resolución de problemas que puedan interferir en la vida personal y /o laboral de los empleados y familiares manteniendo la máxima confidencialidad.

Con los PAE conseguimos:

1. Identificar los problemas en su fase aguda y de este modo la posibilidad de resolución es mayor que si se entra en fase de cronicidad.

2. Que las personas en situaciones de conflicto sepan donde conseguir ayuda.

El programa de prevención y tratamiento de Abuso de Sustancias (Alcohol y Drogas), debería estar implementado en todas las 
Tabla 2. Niveles de intervención en problemas de alcohol desde el Servicio de prevención de riesgos / salud laboral

\begin{tabular}{|c|c|c|c|c|}
\hline $\begin{array}{c}\text { Tipo } \\
\text { intervencion }\end{array}$ & $\begin{array}{l}\text { Poblacion } \\
\text { destinataria }\end{array}$ & $\begin{array}{l}\text { Instrumento } \\
\text { diagnostico }\end{array}$ & $\begin{array}{l}\text { Criterio de } \\
\text { intervencion }\end{array}$ & $\begin{array}{c}\text { Nivel de } \\
\text { intervencion }\end{array}$ \\
\hline $\begin{array}{l}\text { Prevención primaria } \\
\text { Promoción salud }\end{array}$ & $\begin{array}{l}\text { Población laboral total } \\
\text { Colectivos riesgo elevado } \\
\text { Reconocimientos médicos }\end{array}$ & Anamnesis consumo & $\begin{array}{l}\text { Política preventiva de empresa } \\
\text { Abstemios y bebedores: } \\
<21 \text { UBE/smn mujeres } \\
<35 \text { UBE/smn hombres }\end{array}$ & $\begin{array}{l}\text { Asesorar } \\
\text { política empresa } \\
\text { Informar } \\
\text { Formar mandos } \\
\text { Refuerzo hábitos } \\
\text { sanos (modelo). }\end{array}$ \\
\hline $\begin{array}{l}\text { Prevención secundaria } \\
\text { Intervención precoz }\end{array}$ & $\begin{array}{l}\text { Reconocimientos médicos } \\
\text { Consulta individual } \\
\text { o visita oportunista. } \\
\text { Derivación de A.S. } \\
\text { o supervisor }\end{array}$ & $\begin{array}{l}\text { Anamnesis consumo } \\
\text { Marcadores biológicos } \\
\text { Tests diagnósticos } \\
\text { Observ. Supervisor } \\
\text { Evaluación global }\end{array}$ & $\begin{array}{l}\text { Consumo }>21 \text { U mujeres } \\
>35 \text { U hombres por semana } \\
5 \text { U/ocasión (4/mes) } \\
\text { Marcadores positivos o no } \\
\text { AUDIT:> } 8 \text { PUNTOS } \\
\text { CAGE }:<2 \text { puntos } \\
\text { MALT: }<11 \text { puntos }\end{array}$ & $\begin{array}{l}\text { Intervención muy } \\
\text { breve } \\
\text { Consejo aislado } \\
\text { Intervención breve } \\
\text { Informar, Consejo } \\
\text { médico } \\
\text { E. motivacional, } \\
\text { Asesoramiento } \\
\text { Seguimiento. }\end{array}$ \\
\hline $\begin{array}{l}\text { Prevención } \\
\text { secundaria/terciaria }\end{array}$ & $\begin{array}{l}\text { Reconocimientos } \\
\text { médicos } \\
\text { Consulta individual } \\
\text { o visita oportunista } \\
\text { Derivación de A.S. } \\
\text { o supervisor }\end{array}$ & $\begin{array}{l}\text { Anamnesis consumo } \\
\text { Marcadores biológicos } \\
\text { Tests diagnósticos } \\
\text { Observ. Supervisor } \\
\text { Evaluación global }\end{array}$ & $\begin{array}{l}\text { Patología/Marcadores + } \\
\text { (Consumo perjudicial) } \\
\text { CAGE: } 2 \text { puntos } \\
\text { MALT: > } 11 \text { puntos } \\
\text { Dependencia }\end{array}$ & $\begin{array}{l}\text { Desintoxicación } \\
\text { Abstinencia } \\
\text { Derivación "trabajada" } \\
\text { Coordinación } \\
\text { Facilitar cumplimiento } \\
\text { programa externo }\end{array}$ \\
\hline Prevención terciaria & $\begin{array}{l}\text { Alcohólico en tratamiento } \\
\text { o recuperado }\end{array}$ & $\begin{array}{l}\text { Derivación A.S. } \\
\text { o supervisor } \\
\text { Derivación de servicio } \\
\text { externo o devolución } \\
\text { del caso para seguimiento }\end{array}$ & $\begin{array}{l}\text { Rehabilitación en curso } \\
\text { Situación riesgo elevado: } \\
\text { abandono tratamiento, } \\
\text { ansiedad, autosuficiencia }\end{array}$ & $\begin{array}{l}\text { Prevención recaída } \\
\text { (apoyo programa } \\
\text { rehabilitación) } \\
\text { Reinserción laboral } \\
\text { Estudio puesto trabajo }\end{array}$ \\
\hline
\end{tabular}

empresas, ya que el $10-15 \%$ de la población sufre algún tipo de adicción. Estos programas deben estar dirigidos no solo a los trabajadores con problemas relacionados con el alcohol, sino a todos los empleados, ya que la ingesta de bebidas alcohólicas afecta el rendimiento en el trabajo incluso en empleados que no presentan problemas relacionados con el alcohol; esto incluye trabajadores que toman alcohol en la comida y trabajan por la tarde o empleados que toman alcohol por la noche o los fines de semana ${ }^{13}$. Varios estudios han demostrado que el riesgo de accidentes aumenta considerablemente con consumos muy bajos de alcohol y que los esfuerzos preventivos realizados con los bebedores moderados tendrán un resultado más importante en la reducción de accidentes relacionados con el alcohol que los esfuerzos dirigidos a los bebedores importantes ya que son menos ${ }^{17-19}$. El Servicio Médico tiene un importante papel en el desarrollo del Programa de Alcohol ya que:

- Determina la aptitud del empleado para el desempeño de su trabajo.

- Evalua los casos de posible Abuso de sustancias.

- Gestiona y realiza un seguimiento del tratamiento rehabilitador para los empleados que lo precisen.

- Realiza un seguimiento del consumo de sustancias durante el periodo de prueba, tras la incorporación del empleado a su puesto después de haber sido rehabilitado.

- Salvaguarda y garantiza la confidencialidad de la información de los casos. 
- Coordina la formación para la prevención de todos los empleados.

El objetivo del programa de Alcohol y/o Drogas es colaborar en la promoción de la Salud y el bienestar de los empleados y minimizar los problemas laborales que surgen como consecuencia del abuso de alcohol.

Para que tenga éxito, el programa tendrá que contar con el apoyo del equipo directivo de la empresa, ya que cualquier actuación dirigida a que alguien beba menos o deje de beber implica realizar un cambio de hábitos y en ocasiones una adecuación de la persona a su puesto de trabajo con la colaboración de su supervisor.

En un campo tan complejo como el alcoholismo es imprescindible contar con un equipo multidisciplinar y desde la empresa hay que motivar a los empleados con problemas para que realicen un proceso de cambio dinámico que les ayude a conseguir los objetivos marcados.

\section{REFERENCIAS}

(1) Gual A, Mondón S :Detección y tratamiento del alcoholismo en atención primaria. Psiquiatría y Atención Primaria, Abril 2000.

(2) Córdoba R,Altisent R,Aubá J: Curso a distancia de Prevención en Atención Primaria. Programa de Actividades Preventivas y Promoción de la Salud en Atención Primaria (PAPPS). semFYC, 1996.

(3) www.dol.gov/dol/asp/public/programs/drugs/ backgrnd.htm

(4) EDIS-FAD:La incidencia de las drogas en el mundo laboral. FAD, Madrid, 1996.

(5) Sanz González J: Epidemiología del alcoholismo. Medicina del trabajo, Marzo-Abril 1997;113-118.

(6) Rodríguez- Martos Dauer A: Problemas de Alcohol en el ámbito laboral.FAD, 1998.

(7) EDIS-UGT: La incidencia de las drogodependencias en el medio laboral. Depart. Confederal de Serv. Sociales, Madrid, 1987.

(8) Webb, G.R., Redman, S., Hennrikus , D,J., Kelman, G,R., Gibberd,R,W. And Sanson-Fis- her, $\mathrm{R}, \mathrm{W}$. The relationship between high-risk and problem drinking and the occurrence of work injuries and related absences. J. Stud. Alcohol 55: 434-446, 1994.

(9) Mortimer,R.G., Filkins, L.D. and Lower, J.S.Court Procedures for identifying Problem Drinkers. Phase II: Final Report. Prepared for the National Highway Traffic Safety Administration, Publication No. PB 209958, Springfied, Va.: National Technical information Services, 1971.

(10) Genevieve M. Ames,PH.D and Joel W. Grube, PH. D: Alcohol Availability and Workplace Drinking: Mixed Method Analyses. J Stud. Alcohol 60:383-393,1999.

(11) Holt, R,R .Occupational stress . In: Golddberger, L, and Breznitz, S.(Eds) Handbook of stress: Theoretical and Clinical Aspects, New York: Free Press, 1982,pp.419-444.

(12) Gold,D,R., Rogacz, S., Bock, N., Tosteson, T,D., Baum, T.M.,Speizer,F.D. and Czeisler,C.A. Rotating shift work, sleep and accidents related to sleepiness in hospital nurses. Amer. J. Publ. HIth 82:1011-1014,1992.

(13) Richardson, G.S., Miner, J.D. and Czeisler, C.A. Impaired driving performance in shiftworkers: The role of the circadian system in a multifactorial model. Alcohol Drugs Driv. 5,6: 265273,1989-90.

(14) Sánchez Pardo, L .Evaluación de la efectividad de los programas de atención a drogodependientes en la empresa. Madrid: Fundación Mapfre (1994).

(15) Moret Francisco: Los accidentes laborales. Formación de Seguridad Laboral, 140-144, Marzo - Abril 2001.

(16) Genevieve M. Ames, Ph. D., Joel W. Grube, Ph. D., and Roland S. Moore, Ph.D: The Relationship of Drinking and Hangovers to Workplace Problems: An Empirical Study. J. Stud. Alcohol 58:37-47,1997.

(17) Rodríguez- Martos Dauer A: El médico de empresa ante los problemas del alcohol. Medicina del trabajo 6,3, Mayo - Junio 1997 (167- 177).

(18) Megias, E (1996). Prevenir consumos en las empresas. .. ¿exigencias o moda ? Itaca 1(1): 31-50.

(19) Thomas W.Mangione, Ph. D., Jonathan Howland, Ph. D., et al: Employee Drinking Practices and Work Performance. J. Stud. Alcohol 60: 261-270,1999. 